This item was submitted to Loughborough's Research Repository by the author.

Items in Figshare are protected by copyright, with all rights reserved, unless otherwise indicated.

\title{
Numerical investigation of aircraft high-speed runway exit using generalized optimal control
}

PLEASE CITE THE PUBLISHED VERSION

https://doi.org/10.2514/6.2018-0879

\section{PUBLISHER}

(c) American Institute of Aeronautics and Astronautics

\section{VERSION}

AM (Accepted Manuscript)

\section{PUBLISHER STATEMENT}

This work is made available according to the conditions of the Creative Commons Attribution-NonCommercialNoDerivatives 4.0 International (CC BY-NC-ND 4.0) licence. Full details of this licence are available at: https://creativecommons.org/licenses/by-nc-nd/4.0/

\section{LICENCE}

CC BY-NC-ND 4.0

\section{REPOSITORY RECORD}

Huang, Zexin, Matt C. Best, and James A.C. Knowles. 2018. "Numerical Investigation of Aircraft High-speed Runway Exit Using Generalized Optimal Control”. figshare. https://hdl.handle.net/2134/32788. 


\title{
Numerical Investigation of Aircraft High-speed Runway Exit Using Generalized Optimal Control
}

\author{
Zexin Huang ${ }^{1}$, Matt C. Best ${ }^{2}$, and James A.C. Knowles ${ }^{3}$ \\ Department of Aeronautical and Automotive Engineering, University of Loughborough, UK
}

\begin{abstract}
To aim at reducing aircraft turnaround time and improving airport operation efficiency, this paper considers the optimization of aircraft ground manoeuvres such as a high-speed runway exit. The aircraft on the ground is a highly nonlinear dynamical system described by a fully parameterized mathematical model. The full aircraft model used in this paper has been further developed to include combined slip tire model. An iterative simulation-based optimization algorithm known as Generalized Optimal Control is employed to investigate the optimal solution for the control input such as nose-gear steering, main-gear brakes and engine thrust. To achieve different control objectives, the cost function is defined accordingly and then minimized by GOC. The optimization results of GOC will help to explore the safety boundary of ground handling and guide the design of a real-time controller.
\end{abstract}

$\begin{array}{ll}\text { Nomenclature } \\ V_{x}, V_{y}, V_{z} & =\text { aircraft translational velocities along each of the aircraft body axes } \\ W_{x}, W_{y}, W_{z} & =\text { aircraft angular velocities about each of the aircraft body axes } \\ F_{x N}, F_{y N}, F_{z N} & =\text { force elements on the nose gear in the local tire coordinate system } \\ V_{x N}, V_{y N}, V_{z N} & =\text { nose gear velocities in the local tire coordinate system } \\ F_{x R}, F_{y R}, F_{z R} & =\text { force elements on the right gear in the local tire coordinate system } \\ V_{x R}, V_{y R}, V_{z R} & =\text { right gear velocities in the local tire coordinate system } \\ F_{x L}, F_{y L}, F_{z L} & =\text { force elements on the left gear in the local tire coordinate system } \\ V_{x L}, V_{y L}, V_{z L} & =\text { left gear velocities in the local tire coordinate system } \\ F_{x T L}, F_{x T R} & =\text { right and left thrust force parallel to aircraft's x-axis } \\ F_{x A}, F_{y A}, F_{z A} & =\text { aerodynamic force elements at the aerodynamic center of the aircraft } \\ M_{x A}, M_{y A}, M_{z A} & =\text { aerodynamic moment elements at the aerodynamic center of the aircraft } \\ F_{z W} & =\text { weight of the aircraft at the center of gravity } \\ X, Y, Z & =\text { global position of aircraft center of gravity } \\ \psi, \theta, \varphi & =\text { yaw angle, pitch angle and roll angle of the aircraft } \\ V_{x G}, V_{y G}, V_{z G} & =\text { aircraft translational velocities in the ground coordinate system } \\ W_{x G}, W_{y G}, W_{z G} & =\text { aircraft angular velocities in the ground coordinate system } \\ \delta_{z N}, \delta_{z R}, \delta_{z L} & =\text { deflection of each tire in the ground coordinate system } \\ S_{R, L} & =\text { the slip ratio of the right and left gear } \\ \bar{S}_{R, L} & =\text { the normalized slip ratio of the right and left gear } \\ \alpha_{N, R, L} & =\text { the slip angle of the nose, right and left gear } \\ \bar{\alpha}_{R, L} & =\text { the normalized slip angle of the right and left gear } \\ C_{x R, L} & =\text { the longitudinal stiffness of the right and left gear } \\ C_{y R, L} & =\text { the cornering stiffness of the right and left gear } \\ \mu_{x R, L} & =\text { the longitudinal friction coefficient of the right and left gear } \\ \mu_{y R, L} & =\text { the lateral friction coefficient of the right and left gear } \\ \Omega_{R, L} & =\text { the wheel angular velocity of the right and left gear } \\ & \end{array}$

${ }^{1} \mathrm{PhD}$ Student, Dept. of Aeronautical and Automotive Engineering, Loughborough University

${ }^{2}$ Senior lecturer in vehicle dynamics, Dept. of Aeronautical and Automotive Engineering, Loughborough University

${ }^{3}$ Lecturer in dynamics and control, Dept. of Aeronautical and Automotive Engineering, Loughborough University

American Institute of Aeronautics and Astronautics 


\section{Introduction}

According to Statistical Summary of Commercial Jet Airplane Accidents by Boeing [1], 11\% of fatal accidents occurred during manoeuvres on the ground. Even non-fatal ground damage incidents can be quite costly both for airlines, which lose revenue, and for passengers who experience delay and risk physical harm. Although there can be serious economic and safety repercussions from ground collisions at airports, such incidents still occur frequently: 50 accidents were recorded by the International Air Transport Association (IATA) over the period 2010-2014 [2]. Of the ground manoeuvre stages identified, taxiing to and from the runway was the ground phase with the highest incident frequency (almost $40 \%$ of the accidents occurred here). Reducing ground collisions in the future is therefore a key priority for both airports and airline operators globally, something that reliably automated ground manoeuvres could facilitate.

A first advantage of automating aircraft ground manoeuvres would thus be to improve the security and reliability of airport operations under different weather conditions and road conditions. A highly reliable and robust controller would allow more stable and faster ground manoeuvres than a human pilot could achieve. In addition, it comes with associated benefits on the economic aspect. For example, the occupation time of the runway for each aircraft can be minimized so as to increase the airport traffic capacities.

Whilst fly-by-wire systems have been commonly used by aircrafts in flight, aircraft-on-ground control remains very limited. A few researches have focused on aircraft ground dynamics. A fully parameterized 6-DOF aircraft model proposed Rankin et al. [6]. A comprehensive bifurcation analysis was carried out to study the lateral dynamics of the aircraft-on-ground. The boundaries of laterally stability were presented in dependence on operational parameters such as steering angle, thrust level and position of center of gravity. In a more recent study, Rankin et al. presented a general approach to evaluate lateral loads on individual landing gears across an entire operating region [7]. The maximum gear loads are determined for two types of manoeuvres and two different operating weights. A generic parameterized turn was defined in terms of approach velocity and the steering input which was represented by a 'tanh' function. It was assumed that the brakes are applied to achieve a desired velocity before entering the turn and then the turn is initiated with the application of steering after brakes are released. These assumptions replicate typical pilot practice, however the optimality of such an approach has not been quantified.

A few control methods have been proposed and employed to automate aircraft ground manoeuvres. J. Duprez et al. proposed a yaw rate control law based on dynamic inversion and feedback linearization in [3]. A linear controller was designed to constrain aircraft yaw rate to follow a linear reference behavior. This method requires the highly nonlinear tire/ground friction forces to be reduced to saturation-type nonlinearities. As an alternative to dynamic inversion, C. Roos et al. presented a non-standard anti-windup control strategy [4][5]. It was used to control the nose gear steer angle. This proposed method was based on a simplified bicycle model in [4] and a linear parameter variable (LPV) aircraft model in [5], associated with an original approximation of the nonlinear tire forces by saturation-type nonlinearities. It was shown through simulations, that the method is efficient to handle low-speed lateral manoeuvres.

Model-based control strategies greatly depend on the quality of the model. A good model should be able to reflect the system responses accurately. The simplified models used to demonstrate control techniques [3 5] have limitations when considering high lateral accelerations. Hence, the aircraft model considered in this paper is developed from Rankin's 6-DOF model and further developed with the inclusion of a combined-slip tyre model. When braking and steering occur simultaneously, the tire develops a slip angle due to turning and a slip ratio caused by the longitudinal force. Therefore, the normalized total slip is introduced to calculate the total tire force. The total tire force builds up rapidly from zero slip and then reaches its maximum at the optimal slip. As the total slip continues to increase, the tire will saturate and result in the reduction of total tire force. Generally, the application of longitudinal slip tends to reduce the lateral force at a given slip angle condition, and conversely, the application of slip angle tends to reduce the longitudinal force at a given braking condition. The proposed optimization algorithm in this paper can be used to determine the maximum required brake torque that can be applied while the tires can still generate sufficient lateral grip.

Throughout this paper, we aim to find the optimal control inputs with regard to a specified cost function. There are no factitiously predefined patterns for any control inputs. The aircraft behavior is limited by itself as a dynamical system. In addition, we take advantage of a fully parameterized aircraft model that allows direct access to all system states and parameters. Hence, the iterative simulation-based optimization algorithm known as Generalized Optimal Control (GOC) can be employed to identify the optimal control inputs. GOC has been employed effectively for ride and handling optimization in cars by Gordon and Best [8] [9]. For any smoothly nonlinear system, the optimal

control inputs that minimize the user-defined cost function can be determined using GOC. Specifically, time-variant control variables (steering, braking, and thrust) and fixed aircraft parameters (e.g. CG and tire parameters) can be 
optimized simultaneously. Such an approach has recently been used to study collision-avoidance strategies for cars [10]. It established the feasible limit of collision-avoidance behavior of a car and the optimal control input that achieves best performance.

This paper considers a 45-degree runway geometry for the high-speed runway exit manoeuvre. Given a specific initial condition without loss of generality, the optimal control varies with different composition of cost functions. There are two scenarios considered here for the runway exit manoeuvre: the aircraft follows the centerline as closely as possible; the aircraft exits the runway as fast as possible using any paths. A specific cost function needs to be designed for each scenario.

The paper is organized as follows: Section 2 gives full details of the aircraft mathematical model. Section 3 explains the general and specific application of GOC. The optimal control sequences in high-speed runway exit are investigated in Section 4. Finally, the study concludes with some considerations on practical implementation of the runway exit manoeuvre in Section 5 as well as directions for future work.

\section{Aircraft Model}

To develop control strategies for an aircraft automation system, it is essential to develop a mathematical model first. This chapter gives details about the derivation and implementation of the full aircraft model that describes the aircraft's behavior on the ground. This model is developed based on Rankin's model [5] which was initially developed from an industrially validated SIMMECHANICS model [11]. It is therefore taken to be a good representative of real aircraft behaviors.

A tricycle model is considered as the overall frame of the aircraft. The airframe is rigid body with six degrees-offreedom (DOF): three translational DOF and three rotational DOF. Three landing gears are attached to the airframe. Specifically, the two main gears are connected with translational joints (moving in vertical axis only) and the nose gear that steers the aircraft is connected with a cylindrical joint (moving in and rotate around the vertical axis).

The model proposed in this paper is constructed based on the test data in normal operating regions, including nonlinear effects where appropriate, for example, the aerodynamics and tire/ground forces. The chosen parameters and sub-component models represent a mid-sized passenger aircraft. The model parameters can be adapted to represent almost any passenger aircraft.

The aircraft's coordinate system is consistent with the aircraft's principle axes of inertia. The origin is defined at the center of gravity, with the $x$-axis pointing towards the nose of the aircraft along the centerline of the fuselage, the $\mathrm{z}$-axis pointing towards the ground and the y-axis points towards right according to the right-hand rule.

Since this paper considers the optimization of steering, braking and thrust simultaneously, Rankin's model has been further developed to meet the requirement, with an addition of a combined-slip tire model. The brakes are only applied on the main-gears which will cause the difference between the wheel speed and contact-patch speed. Therefore, another two state variables are included in order to represent the main-gears' angular rate.

\section{A. Equations of Motion}

The aircraft Equations of Motion are described by a system of coupled ordinary differential equations (ODEs). These equations are derived from Newton's Second Law by balancing either forces or moments in each degree of freedom [12]. Additionally, they are further linked with relative sub-models, for example the tire model and the aerodynamics model. The aircraft translational and rotational velocities in the local body coordinate system are given as six ODEs [5]:

$$
\begin{array}{rlll}
m\left(\dot{V}_{x}+V_{y} W_{z}-V_{z} W_{y}\right) & = & F_{x T L}+F_{x T R}-F_{x R}-F_{x L}-F_{x N} \cos (\delta)-F_{y N} \sin (\delta)-F_{x A}+F_{z W} \sin (\theta), \\
m\left(\dot{V}_{y}+V_{x} W_{z}-V_{z} W_{x}\right) & = & F_{y R}+F_{y L}+F_{y N} \cos (\delta)-F_{x N} \sin (\delta)+F_{y A}+F_{z W} \sin (\varphi), \\
m\left(\dot{V}_{z}+V_{y} W_{x}-V_{x} W_{y}\right) & = & F_{z W} \cos (\theta) \cos (\varphi)-F_{z R}-F_{z L}-F_{z N}-F_{z A} \\
I_{x x} \dot{W}_{x}-\left(I_{y y}-I_{z z}\right) W_{y} W_{z}= & l_{y L} F_{z L}-l_{y R} F_{z R}-l_{z L} F_{y L}-l_{z R} F_{y R}-l_{z N} F_{y N} \cos (\delta)+l_{z N} F_{x N} \sin (\delta)+ \\
I_{y y} \dot{W}_{y}-\left(I_{z z}-I_{x x}\right) W_{x} W_{z}= & & l_{z A} F_{y A}+M_{x A} \\
& & l_{x N} F_{z N}-l_{z N} F_{x N} \cos (\delta)-l_{z N} F_{y N} \sin (\delta)-l_{x R} F_{z R}-l_{z R} F_{x R}-l_{x L} F_{z L}- \\
I_{z z} \dot{W}_{z}-\left(I_{x x}-I_{y y}\right) W_{x} W_{y}= & l_{z L} F_{x L}+l_{z T} F_{x T R}+l_{z T} F_{x T L}+l_{z A} F_{x A}+l_{x A} F_{z A}+M_{y A} \\
& l_{y R} F_{x R}-l_{y L} F_{x L}-l_{x R} F_{y R}-l_{x L} F_{y L}+l_{x N} F_{y N} \cos (\delta)-l_{x N} F_{x N} \sin (\delta)+ \\
l_{x A} F_{y A}+l_{y T} F_{x T L}-l_{y T} F_{x T R}+M_{z A} .
\end{array}
$$


Rankin's model has been developed for this work by introducing additional two ODEs to describe the two maingears' wheel rotations. The ODEs are derived by balancing the moments of the friction force $F_{x R, L}$ and brake torque $T_{\text {brake }}$ about the wheel's roll axis:

$$
\begin{aligned}
& I_{M G} \dot{W}_{R}=-F_{x R} * R_{\text {wheel }}-T_{\text {brake }}, \\
& I_{M G} \dot{W}_{L}=-F_{x L} * R_{\text {wheel }}-T_{\text {brake }},
\end{aligned}
$$

The aircraft dimensions defined with respect to the aircraft center of gravity (CG) are given in Table 1 . The parameter CG is a percentage measured along the mean aerodynamic chord, from the leading edge to the center of gravity. Throughout this paper, the center of gravity is at a forward position, as is $14 \%$ of the mean aerodynamic chord. In terms of aircraft mass $\mathrm{m}$, a lightweight case $(45420 \mathrm{~kg})$ is considered in which no passengers or cargo are on board but the minimum amount of fuel. The weight of the aircraft (denoted as $F_{z W}=m g$ ) acts at the center of gravity along the z-axis in the ground coordinate system considering the pitch and roll angles. The steering angle (denoted as $\delta$ ) is applied on the nose gear. The orthogonal tire forces (denoted as $F_{x N, R, L}, F_{y N, R, L}$ and $F_{z N, R, L}$ ) are defined at the tire-ground contact patch. The aerodynamic forces (denoted as $F_{x A}, F_{y A}, F_{z A}$ ) and moments (denoted as $\left.M_{x A}, M_{y A}, M_{z A}\right)$ are defined at the aerodynamic center of the aircraft. The thrust forces (denoted as $F_{x T R}$ and $F_{y T R}$ ) are parallel to the $\mathrm{x}$-axis of the aircraft.

To describe the dynamics of an aircraft moving on the ground, it is essential to calculate the position and attitude of the aircraft. Therefore, a ground coordinate system is considered here in which we can do the calculations with reference to a fixed location and orientation in space. The global position is defined as $(X, Y, Z)$ while the attitude is defined by the Euler angles $(\psi, \theta, \varphi)$, where $\psi$ is the yaw angle, $\theta$ the pitch angle and $\varphi$ the roll angle. In addition, the translational velocities and angular velocities in the ground coordinate system are defined as $\left(V_{x G}, V_{y G}, V_{z G}\right)$ and $\left(W_{x G}, W_{y G}, W_{z G}\right)$ respectively. Transformations from the local body coordinate system to the ground coordinate system can be derived by using the standard sequence of rotations given in Philips [13]. Considering the pitch angle and roll angle are relatively small, the velocities in the ground coordinate system are derived with small-angle approximation as follows:

$$
\begin{aligned}
& V_{x G}=V_{x} \cos (\psi)-V_{y} \sin (\psi)+\theta V_{z} \cos (\psi)+\varphi V_{z} \sin (\psi), \\
& V_{y G}=V_{x} \sin (\psi)+V_{y} \cos (\psi)+\theta V_{z} \sin (\psi)+\varphi V_{z} \cos (\psi), \\
& V_{z G}=-\theta V_{x}+\varphi V_{y}+V_{z} \\
& W_{y G}=W_{y}-\varphi W_{z} \\
& W_{x G}=W_{x}+\theta W_{z} \\
& W_{z G}=W_{y} \varphi / \cos (\theta)+W_{z} .
\end{aligned}
$$

Another six ODEs are therefore introduced to calculate aircraft's position and attitude:

$$
\begin{aligned}
& \dot{X}=V_{x G}, \\
& \dot{Y}=V_{y G}, \\
& \dot{Z}=V_{z G}, \\
& \dot{\psi}=W_{z G}, \\
& \dot{\theta}=W_{y G}, \\
& \dot{\varphi}=W_{x G} .
\end{aligned}
$$

The position $(X, Y)$ and yaw angle $\psi$ will be used to plot the trajectory of the aircraft's CG.

Table 1 System parameters and values

\begin{tabular}{lcc}
\hline \hline Symbol & Parameter & Value \\
& Dimensions relative to CG position & \\
$l_{x N}$ & X-distance to the nose gear & $\left(10.186+C G \div 100 \times l_{\text {mac }}\right) \mathrm{m}$ \\
$l_{z N}$ & Z-distance to the nose gear & $2.932 \mathrm{~m}$ \\
$l_{x R}, l_{x L}$ & $\mathrm{X}$-distance to the right/left gear & $\left(2.498-C G \div 100 \times l_{\text {mac }}\right) \mathrm{m}$ \\
$l_{y R}, l_{y L}$ & y-distance to the right/left gear & $3.795 \mathrm{~m}$ \\
$l_{z R}, l_{z L}$ & Z-distance to the right/left gear & $2.932 \mathrm{~m}$ \\
$l_{x A}$ & $\mathrm{X}$-distance to the aerodynamic center & $(0.25-C G \div 100) \times l_{\text {mac }} \mathrm{m}$
\end{tabular}

4

American Institute of Aeronautics and Astronautics 


\begin{tabular}{|c|c|c|}
\hline$l_{z A}$ & z-distance to the aerodynamic center & $0.988 \mathrm{~m}$ \\
\hline$l_{x T}$ & $\mathrm{x}$-distance to the thrust center & $(0.25-C G \div 100) \times l_{m a c} \mathrm{~m}$ \\
\hline$l_{y T R}, l_{y T L}$ & $\mathrm{y}$-distance to the thrust center & $5.755 \mathrm{~m}$ \\
\hline$l_{z T}$ & $\mathrm{z}$-distance to the thrust center & $1.229 \mathrm{~m}$ \\
\hline \multirow[t]{2}{*}{$l_{\text {mac }}$} & Mean aerodynamic chord & $4.194 \mathrm{~m}$ \\
\hline & Wheel parameters & \\
\hline$k_{z N}$ & Stiffness coefficient of the nose tire & $1190 \mathrm{kN} / \mathrm{m}$ \\
\hline$k_{z M}$ & Stiffness coefficient of the main tire & $2777 \mathrm{kN} / \mathrm{m}$ \\
\hline$c_{z N}$ & Damping coefficient of the nose tire & $1000 \mathrm{Ns} / \mathrm{m}$ \\
\hline$c_{z M}$ & Damping coefficient of the main tire & $2886 \mathrm{Ns} / \mathrm{m}$ \\
\hline$c_{r r}$ & Rolling resistance coefficient & 0.02 \\
\hline \multirow[t]{2}{*}{$R_{\text {wheel }}$} & Wheel radius of the main landing gear & $0.64 \mathrm{~m}$ \\
\hline & Moment of Inertia parameters & \\
\hline$I_{x x}$ & Moment of Inertia about $\mathrm{x}$-axis & $913200 \mathrm{~kg} \cdot \mathrm{m}^{2}$ \\
\hline$I_{y y}$ & Moment of Inertia about y-axis & $2548000 \mathrm{~kg} \cdot \mathrm{m}^{2}$ \\
\hline$I_{z z}$ & Moment of Inertia about z-axis & $3335000 \mathrm{~kg} \cdot \mathrm{m}^{2}$ \\
\hline$I_{M G}$ & Moment of inertia of main landing gear & $30.925 \mathrm{~kg} \cdot \mathrm{m}^{2}$ \\
\hline
\end{tabular}

\section{B. Tire model}

At the relatively low velocities experienced by an aircraft on the ground, the nonlinear forces generated by the tires have the dominant effect over aerodynamic forces on the aircraft movement. Hence, a realistic tire model is essential to capture the system's response accurately. The forces generated by the tires are calculated with a tire model developed by the GARTEUR Action Group investigating ground dynamics [21]. Throughout this section a second subscript $\mathrm{N}, \mathrm{R}$ or $\mathrm{L}$ following $\mathrm{x}, \mathrm{y}$ or $\mathrm{z}$ indicates the nose, right or left landing gear local coordinate system with which the velocity or force elements are aligned.

The vertical force component on the tire is modelled as a spring and damper system [15]:

$$
\begin{aligned}
& F_{z N}=-k_{z N} \delta_{z N}+c_{z N} V_{z N}, \\
& F_{z R}=-k_{z R} \delta_{z R}+c_{z R} V_{z R}, \\
& F_{z L}=-k_{z L} \delta_{z L}+c_{z L} V_{z L} .
\end{aligned}
$$

The stiffness coefficients $k_{z^{*}}$ and damping coefficients $c_{Z^{*}}$ are listed in Table 1 . The vertical velocity of each tyre $\left(V_{z N}, V_{z R}, V_{z L}\right)$ can be calculated in terms of the aircraft velocities in the local body coordinate system as follows [5]:

$$
\begin{aligned}
& V_{z N}=V_{z}-l_{x N} W_{y}, \\
& V_{z R}=V_{z}+l_{y R} W_{x}+l_{x R} W_{y}, \\
& V_{z L}=V_{z}-l_{y L} W_{x}+l_{x L} W_{y} .
\end{aligned}
$$

Since we assume that the roll axes of the tires are always parallel to the ground, the deflection of each tire $\left(\delta_{z^{*}}\right)$ can be calculated in terms of the position of the aircraft in the ground coordinate system as follows [5]:

$$
\begin{aligned}
& \delta_{z N}=-l_{z N}-Z+l_{x N} \sin (\theta), \\
& \delta_{z R}=-l_{z R}-Z-l_{x R} \sin (\theta)-l_{y R} \sin (\varphi), \\
& \delta_{z L}=-l_{z L}-Z-l_{x L} \sin (\theta)+l_{y L} \sin (\varphi) .
\end{aligned}
$$

The longitudinal force element on each tire consists of two components: the rolling resistance and the force caused by slip ratio (in the case of braking). At a relatively low speed, rolling resistance is the primary motion resistance force. Rolling resistance occurs due to hysteresis in the material of the tire. As a result, the pressure in the 
leading half of the contact patch is higher than that in the trailing half. Consequently, a horizontal force (rolling resistance) is generated to balance the moments about the roll axis of the tire, which is approximated by [5]:

$$
\begin{aligned}
& F_{r r N}=c_{r r} F_{z N} \cos \left(\alpha_{N}\right), \\
& F_{r r R}=c_{r r} F_{z R} \cos \left(\alpha_{R}\right), \\
& F_{r r L}=c_{r r} F_{z L} \cos \left(\alpha_{L}\right) .
\end{aligned}
$$

Here $\mathrm{Crr}$ is the rolling resistance coefficient which is specified in Table 1. When a rolling tire is subject to a lateral force, the tire will drift to the side. An angle known as slip angle will be generated between the direction of tire heading and the direction of travel. Here $\alpha_{*}$ is the slip angle which is defined in the local tyre coordinate system as:

$$
\begin{aligned}
& \alpha_{N}=\arctan \left(\frac{V_{y N}}{V_{x N}}\right), \\
& \alpha_{R}=\arctan \left(\frac{V_{y R}}{V_{x R}}\right), \\
& \alpha_{L}=\arctan \left(\frac{V_{y L}}{V_{x L}}\right) .
\end{aligned}
$$

Since the brake torque is introduced as a control input, a combined-slip tire model developed by Milliken [14] is employed in this paper. When steering and braking occur simultaneously, the tire develops a slip angle due to turning and a slip ratio caused by the longitudinal force [14]. Therefore, it is necessary to introduce another variable of the normalized combined slip $k_{R, L}$ given by:

$$
\begin{gathered}
k_{R, L}=\sqrt{\bar{S}_{R, L}^{2}+\bar{\alpha}_{R, L}^{2},} \\
\bar{\alpha}_{R, L}=\frac{C_{y R, L} \tan \left(\alpha_{R, L}\right)}{\mu_{y R, L} F_{z R, L}}, \\
\bar{S}_{R, L}=\frac{C_{x, L} S_{R, L}}{\mu_{x R, L} F_{z R, L}}, \\
S_{R, L}=\frac{\Omega_{R, L} R_{w h e e l}-V_{x R, L} \cos \left(\alpha_{R, L}\right)}{V_{x R, L} \cos \left(\alpha_{R, L}\right)},
\end{gathered}
$$

The normalized resultant friction force $F_{R, L}$ and its longitudinal and lateral components are calculated in terms of the normalized lateral force $\bar{F}_{y R, L}$ and the normalized longitudinal force $\bar{F}_{x R, L}$ :

$$
\begin{aligned}
F_{N, R, L} & =\sqrt{\bar{F}_{y R, L}^{2}+\bar{F}_{x R, L}^{2}}, \\
\bar{F}_{y R, L} & =\frac{F_{y R, L}}{\mu_{y R, L} F_{z R, L}}, \\
\bar{F}_{x R, L} & =\frac{F_{x R, L}}{\mu_{x R, L} F_{z R, L}}
\end{aligned}
$$

By the equations above, the normalized resultant force can be calculated in terms of the normalized combinedslip using mathematical curve fitting tools. As an example, Figure 2.1 shows the plot of $F_{R, L} v s k_{R, L}$ generated by Pacejka's "magic formula" [16][17] as follows:

$$
\begin{gathered}
F_{R, L}=D^{\prime} \sin (\gamma), \\
\gamma=C^{\prime} \operatorname{acrtan}\left(B^{\prime} \varepsilon\right), \\
\varepsilon=\left(1-E^{\prime}\right) k_{R, L}+\left(\frac{E^{\prime}}{B^{\prime}}\right) \arctan \left(B^{\prime} k_{R, L}\right),
\end{gathered}
$$

The parameters $B^{\prime}, C^{\prime}, D^{\prime}, E^{\prime}$ can be adapted to represent almost any tyre. For the solid curve representing a typical vehicle tire in Figure 2.1, the parameters are defined as $B^{\prime}=0.714, C^{\prime}=1.40, D^{\prime}=1.00, E^{\prime}=-0.20$.

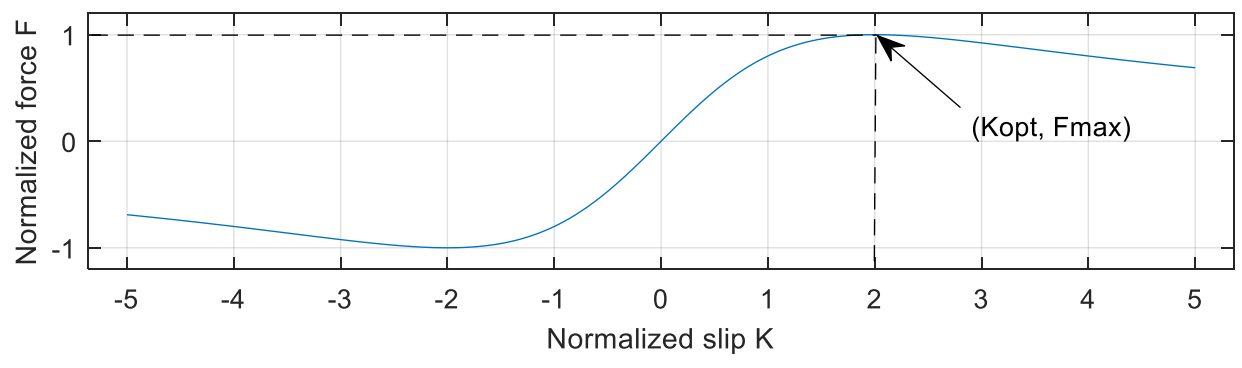

6

American Institute of Aeronautics and Astronautics 
Figure 2.1 The total tire force $F$ is plotted against the normalized slip K. The maximum point Fmax that can be generated by the tire occurs at the optimal slip Kopt.

Additionally, to calculate $\bar{F}_{y R, L}$ and $\bar{F}_{x R, L}$ in terms of the resultant force $F_{R, L}$, the following relationship between them is given as:

$$
\bar{F}_{y R, L}=\frac{\eta_{R, L}\left(k_{R, L}\right) \tan \left(\alpha_{R, L}\right)}{S_{R, L}} \bar{F}_{x R, L}
$$

Here $\eta_{R, L}\left(k_{R, L}\right)$ is a function of slip ratio that makes the above equation hold for both small and large slip angles and slip ratios. The function $\eta_{R, L}\left(k_{R, L}\right)$ is of the form as follows:

$$
\eta_{R, L}\left(k_{N, R, L}\right)=\left\{\begin{array}{cl}
1 / 2\left[1+\eta_{0 R, L}\right]-1 / 2\left[1-\eta_{0 R, L}\right] \cos \left(k_{R, L} / 2\right), & \left|k_{R, L}\right| \leq 2 \pi \\
1 & ,\left|k_{R, L}\right|>2 \pi
\end{array}\right.
$$

where $\eta_{0 R, L}$ is determined from the longitudinal stiffness $C_{x R, L}$, cornering stiffness $C_{y R, L}$, longitudinal friction coefficient $\mu_{x R, L}$, and lateral friction coefficient $\mu_{y R, L}$ :

$$
\eta_{0 R, L}=\frac{C_{y R, L} \mu_{x R, L}}{C_{x R, L} \mu_{y R, L}}
$$

Given the equations above, the normalized longitudinal and lateral forces can be derived as follows:

$$
\begin{aligned}
& \bar{F}_{y R, L}=\eta_{R, L} F_{R, L} \frac{\tan \left(\alpha_{R, L}\right)}{\sqrt{S_{R, L}^{2}+\eta_{R, L}^{2} \tan ^{2}\left(\alpha_{R, L}\right)}}, \\
& \bar{F}_{x R, L}=F_{R, L} \frac{\mathrm{s}_{R, L}}{\sqrt{S_{R, L}^{2}+\eta_{R, L} \tan ^{2}\left(\alpha_{R, L}\right)}},
\end{aligned}
$$

Finally, the actual longitudinal and lateral forces can be derived from the normalized forces as:

$$
\begin{gathered}
F_{x R, L}=\mu_{x R, L} F_{z R, L} \bar{F}_{x R, L}+F_{r r R, L} \\
F_{y R, L}=\mu_{y R, L} F_{z R, L} \bar{F}_{y R, L}
\end{gathered}
$$

\section{Aerodynamic model}

In addition to the tire model, the aerodynamics is another major area where nonlinear behavior can enter the system. The aerodynamic forces are nonlinear since they are proportional to the square of the velocities. Moreover, because of the geometry of the aircraft, the relative aerodynamic coefficients also depend nonlinearly on the angles that the aircraft makes with the airflow: the aerodynamic slip angle $\alpha_{a c}$ and the attack angle $\sigma$. Since we assume that there is no incident wind above the ground, the aerodynamic slip angle can be defined in the same way as the tire model:

$$
\alpha_{a c}=\operatorname{acrtan}\left(\frac{V_{y}}{V_{x}}\right) \text {, }
$$

where $V_{x}$ and $V_{x}$ are the velocities of the aircraft CG in the local body coordinate system. As for the attack angle, it remains relatively steady in the case of ground manoeuvres. The proposed aerodynamics model consists of six elements: three forces and three moments about each axis of the aircraft. It is assumed that all the force elements act at the aerodynamic center of the aircraft, which is defined as the $25 \%$ along the mean aerodynamic chord from its leading edge. The six force elements are modelled as follows:

$$
\begin{aligned}
F_{x A} & =\frac{1}{2} \rho|V|^{2} S_{w} C_{x}\left(\alpha_{a c}\right), \\
F_{y A} & =\frac{1}{2} \rho|V|^{2} S_{w} C_{y}\left(\alpha_{a c}\right), \\
F_{z A} & =\frac{1}{2} \rho|V|^{2} S_{w} C_{z}\left(\alpha_{a c}\right), \\
M_{x A} & =\frac{1}{2} \rho|V|^{2} S_{w} l_{m a c} C_{l}\left(\alpha_{a c}\right), \\
M_{y A} & =\frac{1}{2} \rho|V|^{2} S_{w} l_{m a c} C_{m}\left(\alpha_{a c}\right), \\
M_{z A} & =\frac{1}{2} \rho|V|^{2} S_{w} l_{m a c} C_{n}\left(\alpha_{a c}, \sigma\right),
\end{aligned}
$$

where $|V|$ is the modulus of the aircraft overall velocity, and the parameters $\rho, S_{w}, l_{\text {mac }}$ are listed in Table 1 . The six dimensionless aerodynamic coefficients $C_{x}, C_{y}, C_{z}, C_{l}, C_{m}, C_{n}$ are nonlinear functions of $\alpha_{a c}$. These functions can be obtained from the SIMMECHANICS model developed by the GARTEUR Group [18]. 


\section{Generalized Optimal Control}

In order to develop optimal control strategies for a variety of aircraft ground manoeuvres, the Generalized Optimal Control (GOC) algorithm developed at Loughborough University [8][9] is proposed for this study. In many application scenarios, GOC can be used to identify the optimal control inputs for a particular cost function. This method is based on a Hamiltonian function in terms of system states and co-states (Lagrange multipliers). The optimal control sequence is obtained at the minimum of the Hamiltonian function with respect to control variables.

\section{A. General Formulation}

The GOC algorithm is based on a gradient descent implementation of Pontryagin's Maximum Principle [19] and Marsh [20] for application in dynamical systems. It minimizes a given cost function $\mathbf{J}$ which typically consists of a continuous dynamic cost function $L[x(t), u(t)]$ plus a residual cost $L_{T}[x(T)]$ associated with final states:

$$
J=L_{T}[x(T)]+\int_{0}^{T} L[x(t), u(t)] d t,
$$

A vector of Lagrange multipliers (co-states) $\mathrm{p}(\mathrm{t})$ is introduced and then a Hamiltonian function is defined in terms of the system states and co-states as follows:

where the function $g$ is defined via the system ODEs:

$$
H=L[x(t), u(t)]+p^{T}(t) g[x(t), u(t)],
$$

$$
\dot{x}=g[x(t), u(t)],
$$

The co-states are derived from the following differential equations:

$$
\begin{gathered}
\dot{p}^{T}(t)=-\frac{\partial H}{\partial x}=-\frac{\partial L}{\partial x}-p^{T} \frac{\partial g}{\partial x}, \\
p^{T}(T)=\frac{\partial L_{T}}{\partial x},
\end{gathered}
$$

and the optimal control sequences are found at the minimum of the Hamiltonian function:

$$
\frac{\partial H}{\partial u}=0,
$$

The equations above are all solved over the time interval $[0, \mathrm{~T}]$ under two initial conditions: fixed initial states $x(0)=x_{0}$ and fixed initial co-states $p(T)=p_{T}$. Hence the system is a two-point boundary value problem.

By using the methods given by Marsh [20] and Best and Gordon [8][9], the optimal solution is identified via a discrete sequence of controls and each control element is held constant for an equal time interval $\Delta$ t. Within the time period for each control, the cost gradient is obtained directly from the Hamiltonian as:

$$
\frac{\partial J}{\partial u_{i}}=\int_{t_{i-1}}^{t_{i}} \frac{\partial H}{\partial u_{i}} d t
$$

Therefore, a gradient-based iterative optimization can be utilized to determine the optimal control sequence. Each iteration is conducted in four steps as summarized below. In addition, a flow chart showing the process is depicted in Fig 3.1

Step 1: Calculate the integral of the system state-space from the initial state $x(0)$ using the current discrete control sequence and evaluate the continuous cost $J_{[0, T]}$.

Step 2: Evaluate the residual cost $L_{T}[x(T)]$ in terms of the final state $x(T)$ and hence the initial co-state $p[T]$.

Step 3: Integrate the co-state system via $\partial \mathrm{H} / \partial \mathrm{x}$ in reverse-time from the initial condition $p[T]$ from last step.

Step 4: Calculate cost gradients and hence update the control sequence by a line search optimization along the steepest descent direction (inverse direction of the cost gradient) to minimize $\mathrm{J}$.

Steps 1-4 are repeatedly executed until suitable convergence of cost function is achieved.

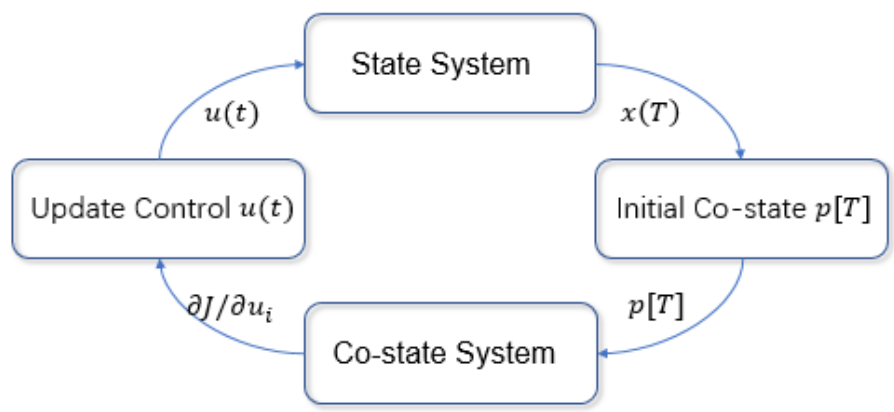

Figure 3.1 Flow chart of the GOC algorithm.

8

American Institute of Aeronautics and Astronautics 


\section{B. GOC Implementation Test: Maximum Deceleration Rate}

The classical $4^{\text {th }}$ order Runge-Kutta method is employed to calculate the time integration of the states and costates. The time step is kept constant throughout the simulation. The state errors are monitored so as to adjust the time step accordingly to ensure reasonable accuracy. Each control unit is a zero-order-hold model for one or multiple time steps. The length of control step will not only affect the fine degree of control, but the convergence speed of cost function as well. Hence, the control time interval should be adjusted accordingly in order to achieve optimality fast and accurately.

The simple scenario of a straight-line deceleration using main gear brakes is considered here. GOC is used to identify the braking required to minimize distance travelled. The initial condition of the aircraft is an equilibrium with the forward speed of $40 \mathrm{~m} / \mathrm{s}$ and the global position of $(X=-1000, Y=0)$. The simulation time is set as 0 to 10 seconds with the time step of $5 \mathrm{~ms}$ which is sufficiently small for this dynamical system. The cost function must be continuous and smooth with respect to all the state and control variables. In this case, the continuous cost function is defined as a track cost $L_{\text {track }}$. To be specific, it is a quadratic function with respect to the aircraft's deviation from the centerline. This continuous cost function makes the aircraft keep a safe distance to the edge of the runway. In addition, a final cost regarding the aircraft final position and orientation ensures that the resulting control strategy is time-optimal, and the aircraft is aligned with the road with no significant yaw rate or sideslip velocity:

$$
L_{\text {final }}=\lambda_{1}\left((X+1000)^{2}+Y^{2}\right)+\lambda_{2} \psi^{2}+\lambda_{3} W_{z}^{2}+\lambda_{4} V_{y}^{2}
$$

The $\lambda$ coefficients are set based on the initial condition and then rescaled to ensure that all the cost components are in the same order of magnitude. The total cost is comprised of the continuous track cost plus the final cost:

$$
L_{\text {total }}=L_{\text {track }}+L_{\text {final }} \text {. }
$$

The final optimal solution is obtained after 2200 iterations as shown in Fig 3.2. The convergence is determined by the cost gradient. The final cost gradients are shown in Fig 3.2(f). Compared with the initial cost gradient, the magnitude has been reduced 10,000 times. Further iterations see only reduction of cost gradients while the total cost remains unchanged which indicates that the cost function has converged. In this case, the total slip consists of longitudinal slip ratio only without lateral slip. The optimal braking torque is shown in Fig 3.2(a). According to the formulation of longitudinal and lateral force on the tire, the maximum deceleration occurs when the total slip equals the optimal slip. Comparing Fig 3.2(a) and Fig 3.2(e), it can be seen that the braking torque varies with the dynamical load on the main gear. Since the optimal slip is an increasing function of the vertical load, the more load on the tire means the more brake torque can be applied before saturation. To achieve the maximum deceleration, the total slip of the tire should stay on the critical level as can be seen in Fig 3.2(c). A very small period of over-slip at the beginning is caused by the inertia of the wheel. A tiny difference between the optimal slip and total slip at the end of the simulation can be seen, though it will be eliminated by continuing the GOC optimisation for enough time. It shows in Fig 3.2(d), however, the normalized tire forces (w.r.t. the maximum force) have already been perfectly controlled at the value of ' 1 ' which means maximum friction force.

Fig 3.2(b) shows the main-gears' wheel speed and contact-patch speed. The difference between them results in the longitudinal slip ratio. When the brake torque is applied, the wheel speed will be reduced. In turn, the linear speed becomes lower than the translational speed which results in a negative slip ratio. Therefore, a negative friction force acts on the main-gears to slow down the aircraft. From the dashed curve of longitudinal velocity, it shows that the aircraft is at a constant deceleration rate of $3 \mathrm{~m} / \mathrm{s}^{2}$, namely the maximum deceleration rate by main-gears' braking.

Fig 3.2(e) shows the dynamic load on the nose gear and the main gear. There is a small weight transfer from the main gears to the nose gear due to the deceleration. The corresponding reduction in brake torque can be seen in Figure 3.2(a). Then the loads on main gear and nose gear increase at the same time. This is because the aircraft speed is slowed down such that the aerodynamic lift decreases.

In conclusion, this simple test case shows that the implementation of GOC is correct and able to optimize the control input to achieve the minimum of a cost function. The tire forces are well controlled just before the saturation to gain the maximum deceleration rate. Furthermore, GOC will be used to investigate more complicated ground manoeuvres. The formulation of the problem and results are discussed in the next section. 

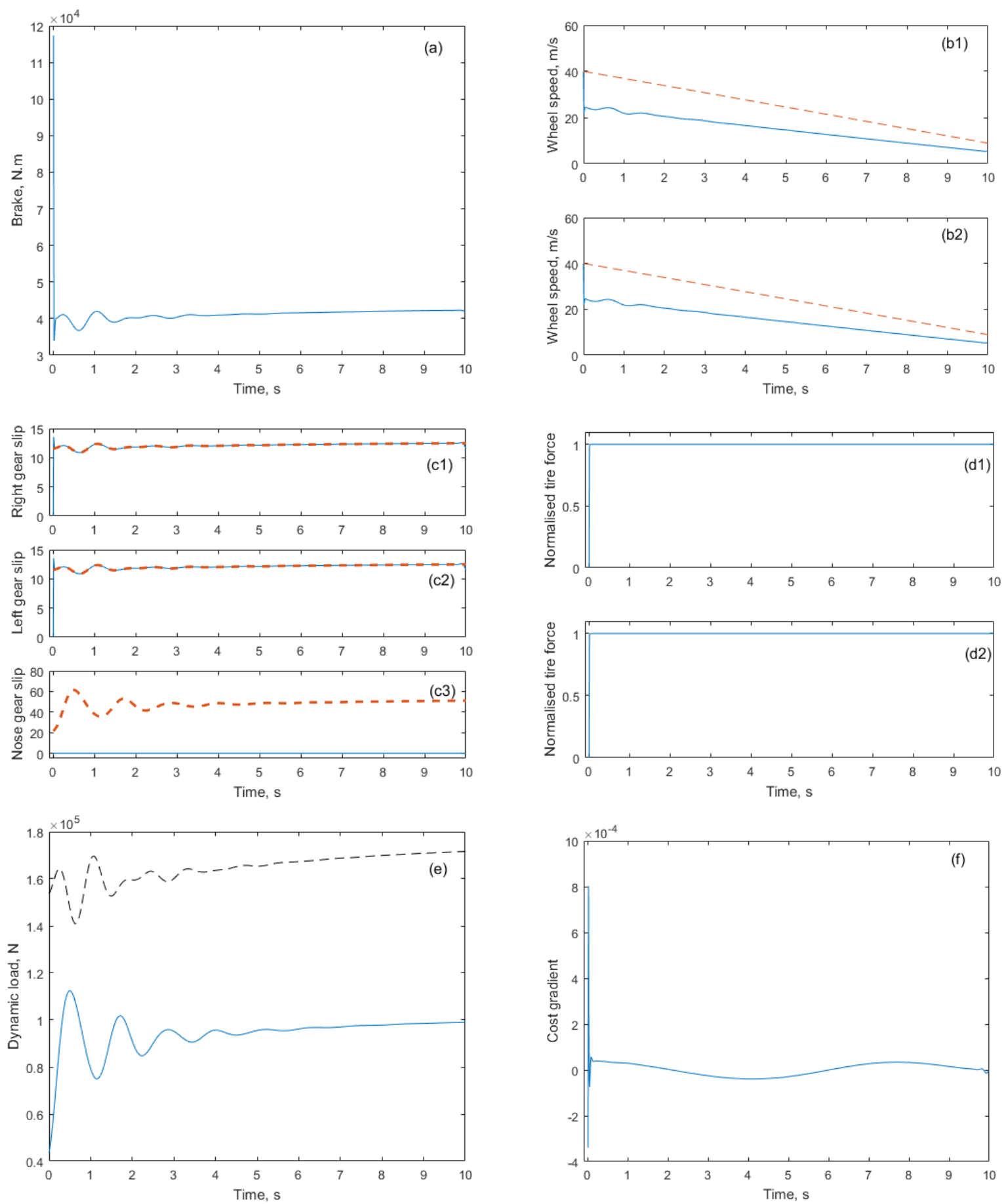

Fig 3.2 A straight-line braking optimization to minimize distance travelled. Panel (a) shows the optimal brake torque input required. Panel (b) shows the wheel speed (solid line) and contact-patch speed (dashed line) for the right (b1) and left (b2) gear. Panel (c) shows the optimal slip (dashed line) and the actual slip (solid line) for the right (c1), left (c2), and nose (c3) gear. Panel (d) shows the normalized friction force for the right (d1) and left (d2) gear. Panel (e) shows the dynamic load on the nose gear (solid line) and main gears (dashed line). Panel (f) shows the cost gradient with respect to brake.

10

American Institute of Aeronautics and Astronautics 


\section{Optimization of High-speed Runway Exit}

In this section, GOC is used to optimize a runway exit manoeuvre. The runway geometry considered here is a 40-meter-wide 45-degree runway exit with two line-segments and one arc-segment, as is shown in Fig 4.1(a). The track cost is defined as a quadratic function of the distance to the runway's centerline. Based on the same runway geometry and the track cost, two cases are considered here: in case 1, the aircraft follows the centerline as closely as possible; in case 2, the aircraft exits as fast as possible.
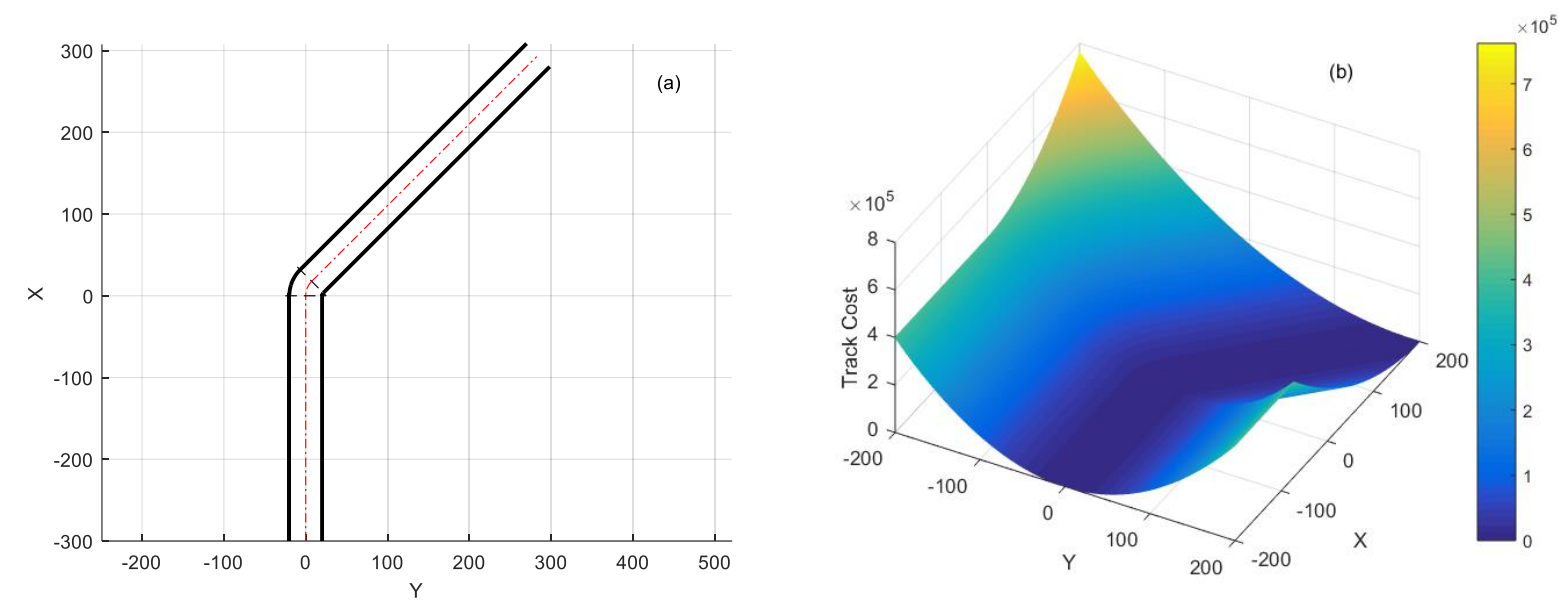

Fig 4.1 Runway and track cost. Panel (a) shows the 45-degree runway exit geometry in the ground X-Y coordinate system. Panel (b) shows the quadratic track cost in terms of the deviation from the centerline.

\section{A: Runway exit following the centerline}

In this case, GOC will control the aircraft to follow the centerline of the runway without considering the final distance to a target point. By doing this, the aircraft will follow the given path and end up with a target yaw angle of 45 degrees. The initial condition of the aircraft is an equilibrium with the forward speed of $40 \mathrm{~m} / \mathrm{s}$ at the global position $(\mathrm{X}=-250, \mathrm{Y}=0)$. The continuous cost function is the track cost as depicted in Fig 4.1(a). The residual cost is a function of the aircraft's yaw angle, yaw rate and lateral velocity, to ensure that the aircraft is aligned with the road with no significant yaw rate or side-slip velocity. Hence, the overall cost function is defined as follows with coefficients $\lambda$ :

$$
\begin{gathered}
L_{\text {final }}=\lambda_{1}(\psi-\pi / 4)^{2}+\lambda_{2} W_{z}^{2}+\lambda_{3} V_{y}^{2}, \\
L_{\text {total }}=L_{\text {track }}+L_{\text {final }} .
\end{gathered}
$$

The cost function converged after 50,000 iterations as shown in Fig 4.2(a). The total cost drops dramatically within the first 1000 iterations and then settles down gradually over 49000 iterations. Since the magnitude of the cost gradients has been reduced by a factor of 10000 as shown in Fig 4.2(b), plus no further reduction of total cost can be achieved with further iterations, the cost function is judged to have converged. The spike on the cost gradient would shrink with further iterations, but it is sufficiently small $\left(O\left(10^{-3}\right)\right)$ to be considered as converged. The final aircraft trajectory is illustrated in Fig 4.2(c) with small triangles representing the aircraft's position and orientation at each second of the simulation. It can be seen that the aircraft has followed the centerline very well with an ideal final state of 45-degree yaw angle.

The aircraft steers to the left when approaching the exit in order to get a larger turning radius. The optimal control inputs of steering, thrust and braking are depicted in Fig 4.3(a). The negative steering angle at the beginning shows that the aircraft is turning to the left initially. However, the inertia of the aircraft is so huge that the aircraft yaw angle builds up after a delay of around 3 seconds. Since the initial forward speed of $40 \mathrm{~m} / \mathrm{s}$ which is too fast to make a 45-degree turn, reverse thrust, and brakes are applied to reduce the aircraft's speed. Fig 4.3(b) shows the wheel speed and contact-patch speed of the right and left gear. It shows that the forward speed drops dramatically from $40 \mathrm{~m} / \mathrm{s}$ to less than $20 \mathrm{~m} / \mathrm{s}$. It can be seen that the right gear wheel speed drops towards zero during peak braking, however, the brake torque is released to a lower level before the right gear's wheel locks. By doing this, the aircraft's dynamics is still under control and the tires can be recovered from the saturation status. 

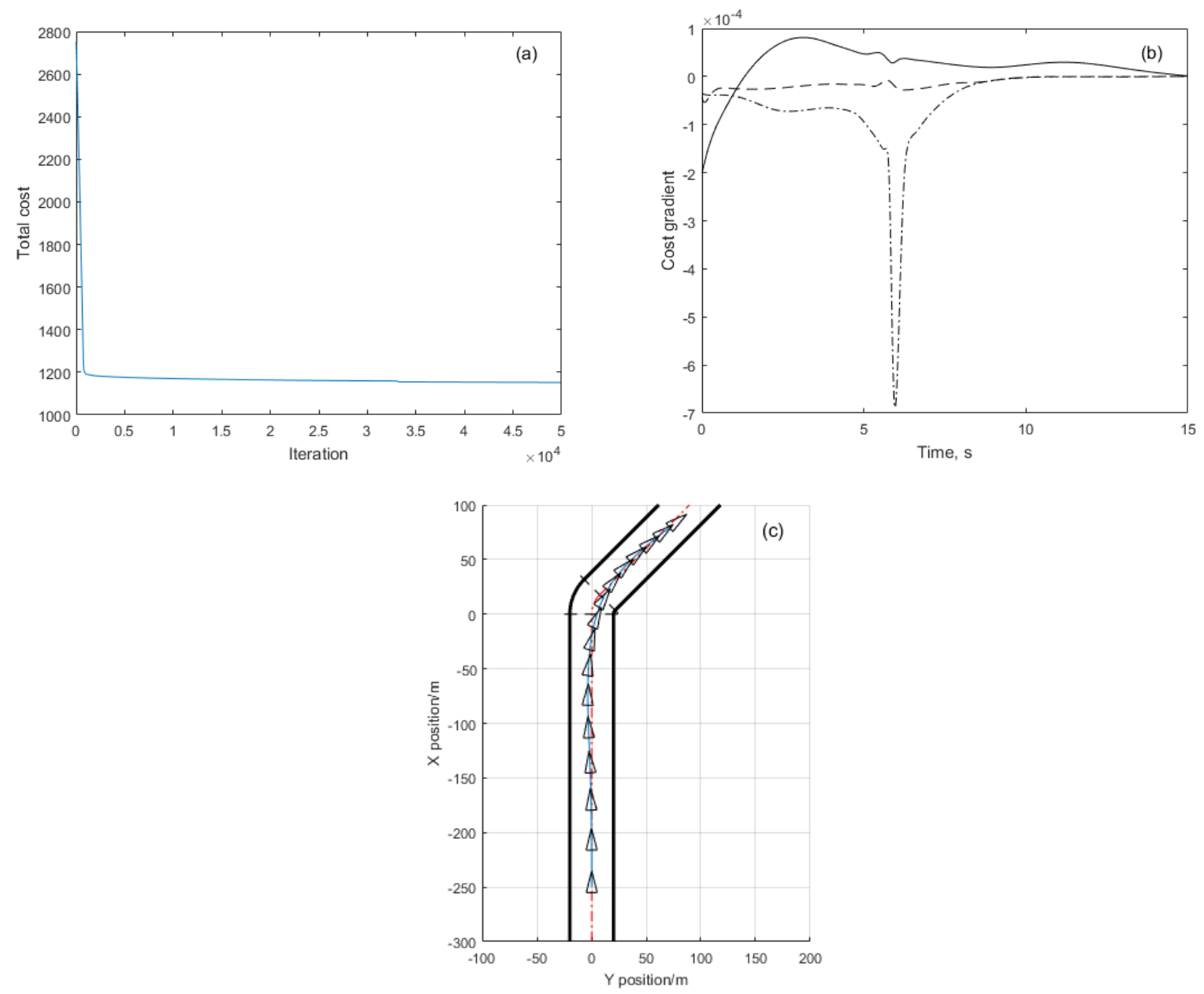

Fig 4.2 Panel (a) shows the total cost along the 50,000 iterations. Panel (b) shows the final cost gradient in terms of steer (solid line), thrust (dashed line), and brake (dashdot line). Panel (c) shows the optimal trajectory of the aircraft CG with small triangles illustrating the aircraft position and orientation at each second of the simulation.

The rapid increase in the braking torque can be seen after $5 \mathrm{~s}$ which results in the right-gear's tire saturation. The slip angles of each tire are depicted in Fig 4.3(c). In the right-gear subplot, the over-slip occurs when the total slip exceeds the optimal slip. In turn, the tire saturation leads to the decrease of the right gear's lateral friction force as shown in Fig 4.3(d). In addition, the total yaw moment around the $\mathrm{z}$ axis is increased due to the reduction of the lateral grip so as to allow the aircraft to make a turn more easily. The optimality is also reflected in Fig 4.3(f), which shows the normalized total friction force on main gears. The forces are normalized with respect to the maximum force that the tire can generate. It can be seen that the normalized force on the right gear stays right on the maximum level during the turn except when it over-slips to make a quick adjustment to the yaw angle. 

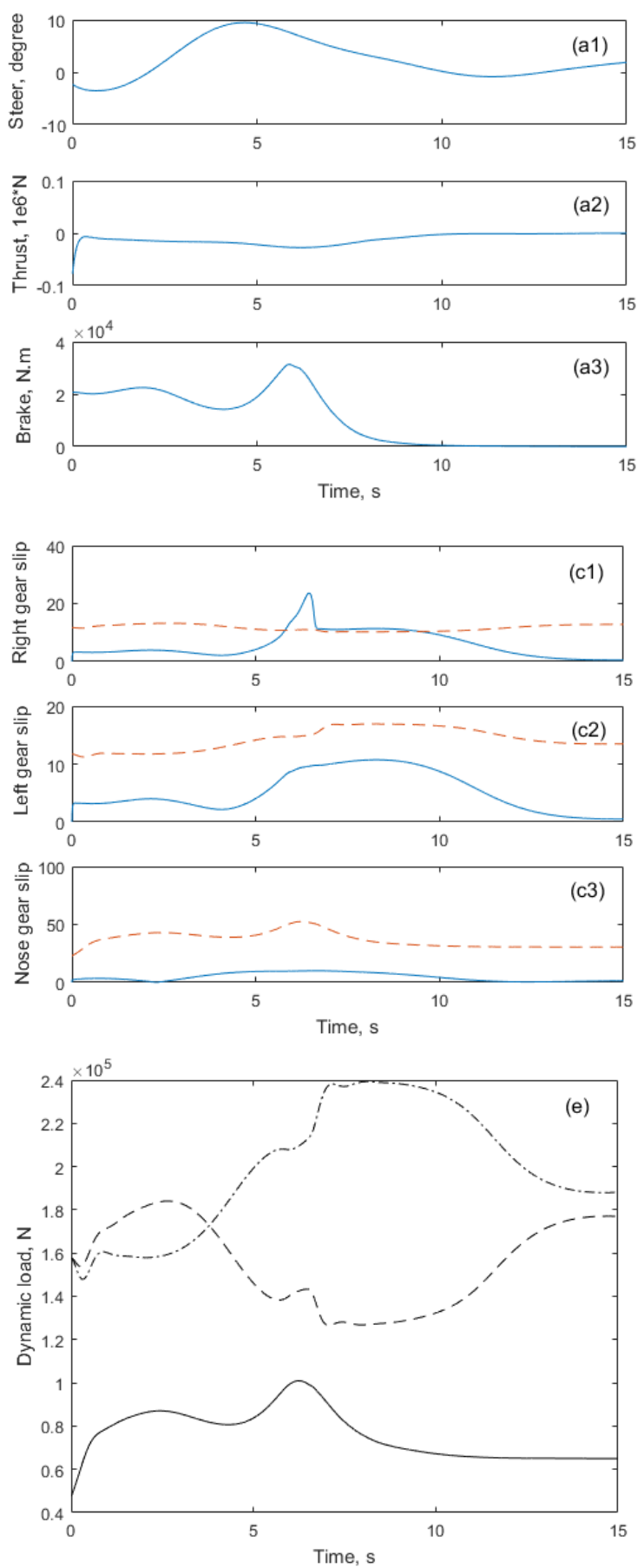

Fig 4.3 Optimal 45-degree runway exit following the centerline. Panel (a) shows the optimal control input of steering (a1), thrust (a2), and braking (a3). Panel (b) shows the wheel speed (solid line) and the contact-patch speed (dashed line) of the right (b1) and left (b2) gear. Panel (c) shows the optimal slip (dashed line) and the actual slip (solid line) of the right (c1), left (c2), and nose (c3) gear. Panel (d) shows the longitudinal (solid line) and lateral (dashed line) force element of the total tire/ground friction force of the right (d1) and left (d2) gear. Panel (e) shows the dynamic load on the nose gear (solid line), right gear (dashed line), and left gear (dashdot line). Panel (f) shows the normalized total tire/ground friction force of the right (f1) and left (f2) gear.

13

American Institute of Aeronautics and Astronautics 


\section{B: Fast exit using brakes and steer}

In this case, a fast 45-degree runway exit manoeuvre is considered. To make comparison with the result in Case 1 , the simulation starts off from the same initial condition (an equilibrium with the forward speed of $40 \mathrm{~m} / \mathrm{s}$ and global position of $(\mathrm{X}=-250, \mathrm{Y}=0)$ and runs for the same period of time from 0 to 15 seconds. The simulation time is set as 0 to 15 seconds with the time step of $10 \mathrm{~ms}$ which is sufficiently small for this dynamic system. The continuous cost function remains the same as in Case 1, which is a quadratic track cost with respect to the deviation from the centerline. This continuous track cost ensures the aircraft moves within the track's boundary. The final cost is defined with respect to the final distance (to an unachievable point to ensure that the resultant control strategy is time-optimal), and the final attitude (to ensure that the aircraft is aligned with the road with no significant yaw rate or side-slip velocity). The final cost function is given below:

$$
L_{\text {final }}=\lambda_{1}(\psi-p i / 4)^{2}+\lambda_{2} W_{z}^{2}+\lambda_{3} V_{y}^{2}+\lambda_{4}\left((X-500)^{2}+\left((Y-500)^{2}\right)\right.
$$

The coefficients $\lambda$ are set based on the initial condition and then rescaled to ensure that all the cost components are in the same order of magnitude. $\lambda_{1}, \lambda_{2}, \lambda_{3}$ remain the same as in Case 1. The total cost is comprised of the continuous track cost plus the final cost:

$$
L_{\text {total }}=L_{\text {track }}+L_{\text {final }}
$$
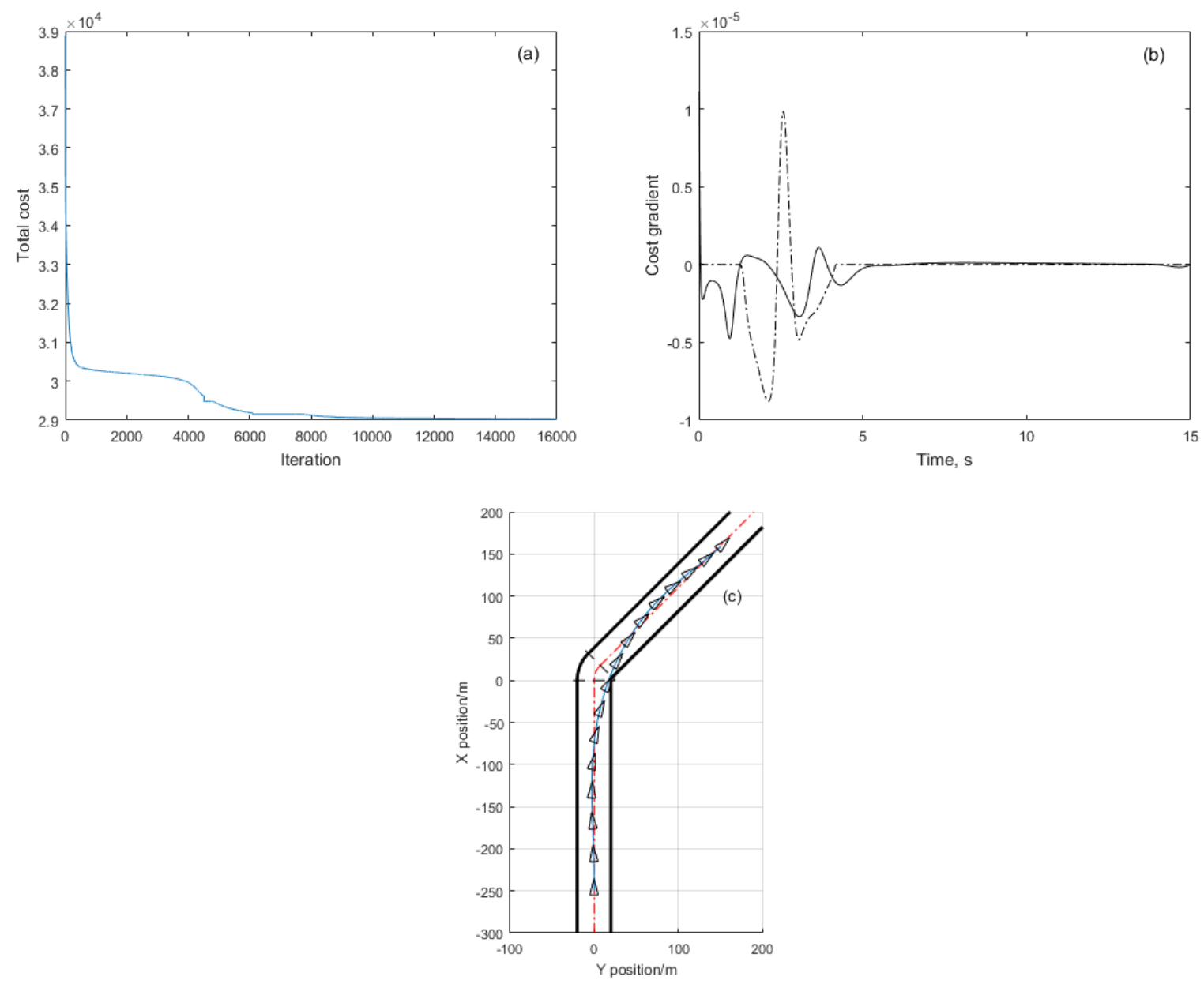

Fig 4.4 Panel (a) shows the total cost along the 16,000 iterations. Panel (b) shows the final cost gradient in terms of steer (solid line) and brake (dashdot line). Panel (c) shows the optimal trajectory of the aircraft CG with small triangles illustrating the aircraft position and orientation at each second of the simulation.

14

American Institute of Aeronautics and Astronautics 
The cost function converges after 16,000 iterations as shown in Fig 4.4(a). The cost drops significantly in the first few hundred of iterations and then settles down gradually over about 9000 iterations. The convergence can be confirmed since the magnitude of the cost gradients has been reduced by factor of 1000 as shown in Fig 4.4(b), plus no further reduction of total cost can be achieved with more iterations. The final aircraft trajectory is illustrated in Fig 4.4(c). Each small triangle represents the aircraft's position and orientation at each second of the simulation.
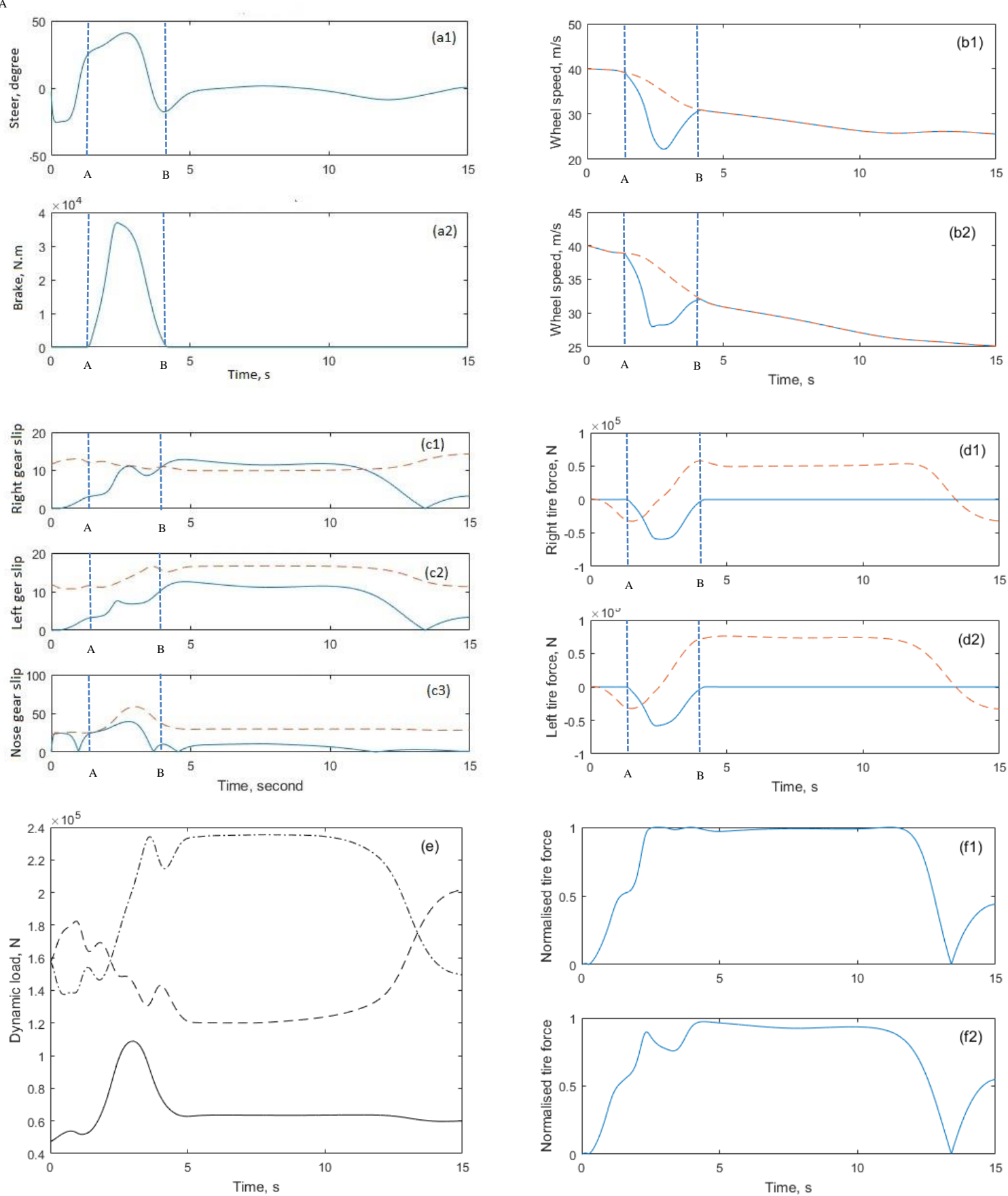

Fig 4.5 Fast 45-degree runway exit: Panel (a) shows the optimal control input of steering and braking. Panel (b) shows the wheel speed (solid line) and the contact-patch speed (dashed line) of the right (b1) and left (b2) gear. Panel (c) shows the optimal slip (dashed line) and the actual slip (solid line) of the nose, right and left gear. Panel (d) shows the longitudinal (solid line) and lateral (dashed line) force element of the total tire/ground friction force of the right (d1) and left (d2) gear. Panel (e) shows the dynamic load on the nose (solid line), right (dashed line) and left (dashdot line) gear. Panel (f) shows the normalized total tire/ground friction force of the right (f1) and left (f2) gear. 
The final optimal solution and several critical intermediate states are illustrated in Fig 4.5. The optimal control sequence of steering and braking are shown in Fig 4.5(a). It can be seen that the aircraft steers to the left initially (negative steering angle) to obtain a larger turning radius, and then to the right to cut the coner. It takes a couple of seconds to build up the aircraft yaw angle due to its huge yaw moment of inertia. The brake is applied initially at point ' $A$ ' to slow down the aircraft when the lateral slip is relatively small. Then just before the aircraft starts to cut the corner, the brake is completely cut off at point ' $\mathrm{B}$ ' to achieve the maximum lateral slip angle. As can be seen in Fig 4.5(c), the solid curve illustrates the total slip and the dashed curve illustrates the optimal slip where the maximum force occurs. The total slip on all the three gears achieved the optimal slip at some point along the manoeuvre which means the maximum tire force was generated. This can be seen clearly in Fig 4.5(f), the normalized tire force reaches a value of ' 1 ', which indicates the tire is operating at its maximum capacity. Due to the weight transfer from right gear to left gear as depicted in Fig 4.5(e), the right gear saturates a little bit to obtain a greater tire force on the left gear such that the total lateral force is maximized.

\section{Conclusion}

From the optimization result of the high-speed runway exit manoeuvre as discussed above, GOC has been revealed to be a powerful tool of optimal control, especially for nonlinear system. GOC can be used to explore the limit of aircraft ground handling by minimizing the cost function. Therefore, the cost function should be defined according to the control objective. All the cost components must be well balanced in case the optimization is stuck due to some extremely high cost. The final optimal control for the 45-degree runway exit might be in a different pattern because of the different initial condition to start with like CG position and velocity. Following simulation experiments will be conducted to investigate a full range of initial velocities with various distances to the exit. Hopefully, the boundary of initial condition can be identified such that the aircraft can cut the corner if only the initial condition is within that boundary. Even though GOC is not suitable for real-time implementation, the optimal solution given by GOC is valuable to guide the design of a closed-loop controller in the future. 


\section{References}

[1] Statistical Summary of Commercial Jet Airplane Accidents, Aviation Safety Boeing Commercial Airplanes P.O. Box 3707 M/C 0A-19 Seattle, Washington 98124-2207 USA.

[2] IATA, “Safety Report 2014”, 51st ed, April 2015, Montreal, ISBN 978-92-9252-582-8.

[3] J. Duprez, F. Mora-Camino, F. Villaume, Aircraft-on-ground lateral control for low speed manoeuvers, in: Proceedings of the 16th IFAC Symposium on Automatic Control in Aerospace, St. Petersburg, Russia, 2004.

[4] C. Roos, J. Biannic, S. Tarbouriech, C. Prieur, M. Jeanneau, On-ground aircraft control design using a parameter-varying anti-windup approach, Aerospace Science and Technology 14 (2010) 459-471

[5] C. Roos, J. Biannic, S. Tarbouriech, C. Prieur, On-ground aircraft control design using an LPV anti-windup approach. In: Bates D, Hagstro"m M, editors. Nonlinear analysis and synthesis techniques for aircraft control, Vol. 365. Berlin Heidelberg: Springer Berlin Heidelberg; 2007. p. 117-45.

[6] J. Rankin, E. Coetzee, B. Krauskopf, M. Lowenberg, Bifurcation and Stability Analysis of Aircraft Turning on the Ground, Journal of Guidance, Control, and Dynamics, Vol. 32, No. 2, March-April 2009

[7] J. Rankin, E. Coetzee, B. Krauskopf, M. Lowenberg, Operational Parameter Study of Aircraft Dynamics on the Ground, Journal of Computational and Nonlinear Dynamics, April 2010, Vol. 5/021007-1.

[8] Gordon, T.J. and Best, M.C. (2002) 'A Sequential Dual Model Approach to Lap Optimisation', Proceedings of the 6th International Symposium on Advanced Vehicle Control (AVEC), Hiroshima, Japan, pp.99-104.

[9] Gordon, T.J. and Best, M.C. (2007) 'On the synthesis of driver inputs for the simulation of closed-loop handling manoeuvres', International Journal of Vehicle Design, Vol. 40, Nos. 1-3, pp.52-76.

[10] Best, M.C. (2012) 'Optimisation of high-speed crash avoidance in autonomous vehicles', Int. J. Vehicle Autonomous Systems, Vol. 10, No. 4, pp.337-354.

[11] MathWorks, 2004, "Model and Simulate Mechanical Systems With SIMMECHANICS," http://www.mathworks.com/products/simmechanics/

[12] B. Etkin, Dynamics of atmospheric flight, Wiley, 1972.

[13] W. F. Phillips, Mechanics of flight, Wiley, January 2004.

[14] W. F. Milliken, D. L. Milliken, "Race Car Vehicle Dynamics", 1995, ISBN 1-56091-526-9.

[15] M. Blundell and D. Harty, The multibody systems approach to vehicle dynamics, SAE International, September 2004.

[16] Bakker, E., L. Nyborg, and H. B. Pacejka, "Tire Modelling for Use in Vehicles Studies", SAE Paper No. 870421, Society for Automotive Engineers, Warrendale, PA, 1987.

[17] Bakker, E., H. B. Pacejka, and L.Lidner, “A New Tire Model with an Application in Vehicle Dynamics Studies", SAE Paper No. 890087, Society of Automotive Engineers, Warrendale, PA, 1989.

[18] M. Jeanneau, Description of aircraft ground dynamics, Garteur FM AG17 RP0412731, GARTEUR, 2004.

[19] Bryson, A.E. and Ho, Y.C. (1975) Applied Optimal Control: Optimisation, Estimation and Control, Hemisphere, New York.

[20] Marsh, C. (1992) 'A Nonlinear Control Design Methodology for Computer-controlled Vehicle Suspension Systems', PhD Thesis, Loughborough University.

[21] Jeanneau, M., 2004, "Description of Aircraft Ground Dynamics," GARTEUR FM AG17, Paper No. RP0412731. 